\title{
Symptoms of Oral Submucosal Fibrosis: Our Experience with Intralesional Injection Treatment
}

\author{
${ }^{1}$ Mudit Mittal, ${ }^{2}$ Rajesh Yadav, ${ }^{3} \mathrm{PV}$ Dhond, ${ }^{4}$ Shashi Kant \\ ${ }^{1}$ Surgeon, Department of Otorhinolaryngology and Head and Neck Surgery, SNLP Cancer Hospital and Research Center \\ Rajkot, Gujarat, India \\ ${ }^{2}$ Resident, Department of Otorhinolaryngology, Bhagwati Hospital, Borivali (W), Mumbai, Maharashtra, India \\ ${ }^{3}$ Head, Department of Otorhinolaryngology, Bhagwati Hospital, Borivali (W), Mumbai, Maharashtra, India \\ ${ }^{4}$ Lecturer, Department of Otorhinolaryngology, KEM, Mumbai, Maharashtra, India
}

Correspondence: Mudit Mittal, Surgeon, Department of Otorhinolaryngology and Head and Neck Surgery, SNLP Cancer Hospital and Research Center, Rajkot, Gujarat, India, Phone: 9558300785, 9425945542, e-mail: dr_muditmittal @yahoo.co.in

\begin{abstract}
Objective: This study evaluates the symptoms and effects of peroral intralesional injections in relieving the symptoms of oral submucosal fibrosis (OSMF) in our clinical settings.

Study design: Retrospective clinical review at a primary care hospital of 32 patients with extensive OSMF who underwent peroral intralesional injections of steroid, hyaluronidase, placentrex and lignocaine performed in office setting

Results: The main symptoms were change in color of buccal mucosa, trismus, burning mouth, vesicles in oral cavity. All patients experienced considerable improvement in their symptoms over a duration of 2 to 6 weeks.

Conclusions: Though, a large number of Indian population is suffering and seeking treatment for OSMF, unfortunately not much has been done in this area. It is difficult to find studies on peroral intralesional injection technique. When there is lack of reliable evidence of oral submucosal fibrosis treatment, the old technique of peroral intralesion shots of steroid, hyaluronidase, placentrex in lignocaine is safe and effective in resolving the symptoms associated with OSMF. The therapy is very cost effective and also reduces the need of surgery.

Keywords: OSMF, Symptoms, Peroral injections.
\end{abstract}

\section{INTRODUCTION}

The condition of oral submucosal fibrosis was first described in India by Joshi in 1953. The disease is prevalent in Indian subcontinent, and its prevalence rate varies from 2 to 5 per 1000. ${ }^{1}$

The most common symptoms are:

1. Burning sensation in oral cavity, especially while chewing hot and spicy food, this is the most bothering symptom also. Sometimes pain is referred to ears and is so severe that patients loose interest in food.

2. Change in color of buccal mucosa, palatine arch and retromolar trigone (mostly leukoplakia).

3. Recurrent painful vesicle formation over buccal mucosa, soft palate or palatine arch.

4. Trismus, sometimes is so severe that patient has only one finger mouth opening. Patient has to shift on semisolid, cold, nonspicy diet often.

5. Difficulty in protruding tongue: Though this symptom has been mentioned in many textbooks and journals, we have not come across any of the patient complaining this during our study.

Although, the exact pathophysiology of OSMF is not known, it appears that some form of prolonged local irritation phenomenon, like habit of chewing betel nut alone or as an ingredient of pan, gutkha or pan masala [a very popular addiction in India which is a combination of betel nut (areca), slacked lime, arecolin with chewing tobacco] is responsible for this. ${ }^{1}$

India, the third largest producer of tobacco in the world, amassed 1.7 million disability-adjusted life years (DALYs) in 1990 due to disease and injury attributable to tobacco use in a population where $65 \%$ of men and $38 \%$ of women consume tobacco. Tobacco consumption continues to grow in India at 2 to $3 \%$ per annum. ${ }^{2}$ With this alarming rate of increasing people consuming tobacco and betel nut, soon the number of patients seeking treatment for OSMF will increase tremendously. Many remedies have been tried for management of OSMF in India and abroad before also, but there has been lack of literature and reliable evidence for the effectiveness of any specific interventions for it.

The current study documents the utility of peroral intralesional injections in managing OSMF patients.

\section{MATERIALS AND METHODS}

Around 32 patients with extensive oral submucosal fibrosis were treated with peroral intralesional injection of Hyaluronidase 50U, 2cc Placentrex, 1cc (4 mg) Dexamethasone mixed in 1 cc of lignocaine $2 \%$, followed by local application of steroid gel and diet modification. 
Pre- and post-treatment examination were performed under Bull's lamp. All patients received peroral injections under head light illumination in office setting after lignocaine sensitivity. The needle was inserted after palpating the bands in buccal mucosa, in the area of leukoplakia, palatine arch, retromolar trigon and those areas where the patients felt most severe burning sensations, and patients were allowed to go home after the procedure. Injections were repeated every week for 6 weeks. All the patients had to stop any form of betel nut, tobacco chewing or smoking before receiving this therapy. Patients were also advised to abstain from hot and spicy food during the course of treatment. Those patients who had restricted mouth opening were advised to do mouth opening exercises also.

After one month of completion of therapy, Mallampati scale was used for grading mouth opening, also every patient was asked to fill a questionnaire to grade the therapy and their overall satisfaction.

\section{RESULTS}

Around $81.25 \%$ patients presented in our OPD with OSMF were male and only $15.6 \%$ were female. All the 6 females were in their late thirties. None of them had marked trismus but burning sensation in mouth and change in color of oral mucosa were their most common complaints.

Around 31 patients presented in our OPD with OSMF, belonged to age group 20 to 40 years. A 21-year-old male had marked trismus (class IV on Mallampati scale) and was unable to pass even single finger in his mouth. It was difficult to palpate the bands to give intraoral injections, but after 6 weeks of cocktail therapy and mouth opening exercises his mouth opening improved to two fingers (class III Mallampati scale).

The mean duration of symptoms is 11.65 months. Four patients were tobacco addicted since 2 years, one since 20 years and rest of them since few months only. The patients also complained that even after stopping tobacco chewing still there is progression of symptoms. The mean duration of partial/complete resolution of symptoms is 4.75 weeks. The patients were advised to adhere to the plan and complete their full 6 weeks therapy.

Burning sensation in mouth was the complaint of all the patients seeking treatment. Out of 32 patients who had burning sensation in their mouth, 30 of them had relief from this symptom, 2 patients reported to have partial relief only.

All the 32 patients in our study had discoloration of their buccal mucosa due to OSMF. After the completion of treatment, only 20 patients had noticeable change in color (back to pink), rest 12 did not feel any significant change. 10 out of 32 patients had tendency to develop painful vesicles frequently in mouth. During the treatment and till the date of writing this paper none of them reported to have vesicles again (Fig. 1).

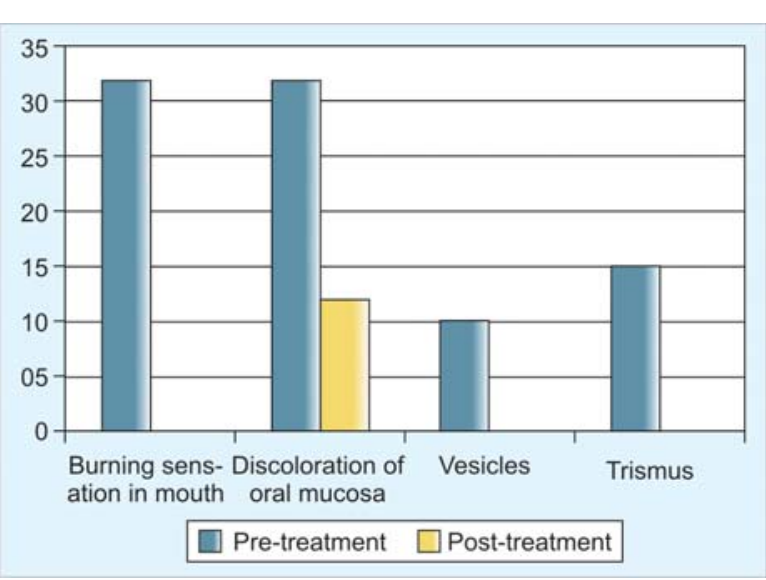

Fig. 1: Pre- and post-treatment evaluation of OSMF patients

For mouth opening, Mallampati scale was used. Around 15 patients had trismus either class III or IV on Mallampati scale. Post-treatment of all the 15 patients had improvement of at least one class on this scale.

In our study, 29 patients had an average of $97.5 \%$ satisfaction. Improvement was observed in as little as 2 weeks.

\section{DISCUSSION}

The male to female ratio of patients of OSMF is $4.3: 1$ in this study, confirms the preponderance of male patients suffering from OSMF. It can be due to the tobacco and betel nut chewing habit in India, which is more common in males.

In this study, 96.8\% were between 20 and 40 years. Ahmad MS et al had a similar observation in their study, maximum number of cases belonged to 21 to 40 years of age. ${ }^{3}$ The study conducted by Naresh R Makwana et al also emphasizes the fact that, majority of addicted adolescents was in the age group of 17 to 19 years (36.26\%). Tobacco chewing is the most frequent form of using tobacco by adolescents than smoking. ${ }^{4}$ This coincides with the age of presenting symptoms of OSMF in our study which starts within 1 or 2 years of addiction.

As said earlier, though the exact pathophysiology of OSMF is not known, betel nut and tobacco are supposed to be responsible for it. It has also been shown in many studies before that the gutkha was most commonly used by the OSMF cases. Only 3\% did not use any gutkha or other areca nut product. ${ }^{3}$ The nut causes mechanical irritation because of its hard and rough surface or chemical irritation because of arecoline, chewing tobacco and smoking. Thus, it is a chronic insidious process characterized by juxta-epithelial deposition of fibrous tissues in oral cavity. ${ }^{1}$

Thangjam GS et al in their study found that transglutaminase-2 (TGM-2) over expression in oral submucous fibrosis (OSMF) and its regulation by arecoline in oral fibroblasts is one of the causative factors for OSMF. ${ }^{5}$ 
Another research establishes relation that arecoline and oral keratinocytes may affect the collagen metabolism of fibroblasts and play important role in pathogenesis of OSMF. $^{6}$

Chronic irritation brings a fibroelastic transformation of connective tissue in lamina propria associated with epithelial atrophy, sometimes preceded with vesical formation. In later stages, the fibrosis is marked, there is progressive trismus. ${ }^{7}$

In current therapy, injection Hyaluronidase and Placentrex with steroid have been used locally, no previous use in this manner has been found in any of the studies for OSMF even after meticulous search by the authors.

Human placental extracts have been used by many clinicians for the same purpose since long but nothing much is available in literature. Kaushal V et al have studied its effects on radiation induced mucositis and reported that human placental extract appears to be effective in the management of radiation-induced oral/oropharyngeal mucositis and especially in controlling subjective symptoms. ${ }^{8}$ They had their trial with intramuscular injections of Placentrex.

Hyaluronidases depolymerize and hydrolyze the hyaluronic acid component, with resulting increase in spread. Subsequently, the hyaluronic acid is replaced or restored to its original state, and the barrier to spread is reconstituted. ${ }^{9}$ Hyaluronidase is a spreading or diffusing substance, which modifies the permeability of connective tissue through the hydrolysis of hyaluronic acid, a polysaccharide found in the intercellular ground substance of connective tissue; it temporarily decreases the viscosity of the cellular cement and promotes diffusion of injected fluids or of localized transudates or exudates, thus facilitating their absorption.

All 32 patients came to OPD with complaints of burning sensation in oral cavity. This was most debilitating problem but after full course of cocktail therapy all the patients had relief from this problem. Most of the patients once again started their normal regular diet. Sinha et al achieved relief in $100 \%$ cases by injection Hydrocortisone in comparison with $90.9 \%$ cases by injection Placentrex. ${ }^{10}$ Moreover, it was a 12-week therapy, and if one of their therapies was not found effective then they switched over to the other. In our study, two patients did not stop chewing tobacco but still they had relief in this symptom. As far as change in color of buccal mucosa is concerned, only $62.5 \%$ found significant change in color of oral mucosa, again back to pink. Here, Sinha et al in their study comparing Hydrocortisone and Placentrex found that the pink color of mucosa was regained in $94.2 \%$ of cases treated with injection Hydrocortisone whereas it was regained in only $66.6 \%$ of cases with injection Placentrex.

Placentre ${ }^{10}$ can be drawback of this therapy, as we found that the change in color of buccal mucosa is most resistant to respond to any therapy and cannot be brought to normal in all of the patients; as some of the patients with tobacco addiction continue chewing tobacco. None of the patients in our study developed any vesicles again during the course of therapy till the writing of this research paper. $100 \%$ patients had relief in their complaint of difficulty in mouth opening. Sinha et al recorded relief in $92.8 \%$ of cases, with injection Hydrocortisone whereas it improved in only $68.7 \%$ cases with injection Placentrex. ${ }^{10}$

Histopathologically, the effects of injection Hydrocortisone and injection Placentrex on buccal mucosa was:

1. The epithelium which was stratified, squamous remained unaffected.

2. Extensive fibrosis in submucous space was reduced after therapy.

3. Keratinization and chronic inflammatory cells decreased.

4. Blood vessels were found dilated.

5. Formation of new capillaries were seen.

6. Glandular tissue was seen which was absent prior to therapy. ${ }^{10}$

What is compelling about this study is that all the patients with OSMF symptoms, responded with this therapy very well. The average satisfaction grade was $97.5 \%$. Therefore, whatsoever the etiology of OSMF is, this therapy merits consideration, especially when other methods have failed.

Lack of satisfaction in three patients could also explain the expectation of the patient from the cocktail therapy. Important fact to note here is that two of these patients could not help themselves abstaining from gutkha chewing. Possibility of long-standing complaints and some psychiatric association cannot be denied. Obviously, the need and the expectations of the patient to be weighed against the risks and benefits of any form of therapy.

The most interesting fact about this study is that all patients experienced resolution of most of their symptoms, specially the most troublesome problem of burning sensation in mouth, which has been keeping them away from warm and slightest of spicy food. In India, people have habit of hot and spicy food compared to the food habit of western world. Due to this problem, patients loose taste and interest in food.

The time invested in cocktail therapy must be weighed against the time needed for surgery and postoperative period with potential long-term complications for surgery. Lack of reliability of effectiveness of single drug at a time, which is also time consuming and if patients do not respond to one, switching to another drug needs compliance from patient, which is not possible in Indian circumstances.

Monitoring the patients following the injections may be important in order to assess their response to this therapy and hypersensitivity reaction developing, if any. In such conditions, further use should be contemplated.

Twenty out of 32 patients had taken some medications for OSMF before starting this therapy at some point of time and did not get any relief. They developed a firm belief that 
they have to live rest of their life with this problem. Six out of 32 were also advised surgery for marked trismus but they refused for surgery and opted for intralesional injection therapy.

At this place, per week therapy cost was US \$3 and the drugs were injected in office setting. This is not an inconsequential consideration when compared with the cost of other treatment modalities (surgery or forceful mouth opening under anesthesia) and suffering of the patients. A therapy which may offer enhanced cure rate must be considered within our armamentarium of treatment option. Overall, the intralesional injection represents a cost effective way to resolve sufferings of OSMF patients.

Globalization has brought people together and diseases as well. Though this disease mainly affects Indians, people of Indian origin living abroad are not untouched by it.

\section{CONCLUSIONS}

- The management of OSMF with intralesional injection is effective in resolving the symptoms associated with OSMF. The therapy is very cost effective and can sometimes obviate the need of surgery also.

- Change in color of buccal mucosa is most resistant symptom to the treatment.

\section{REFERENCES}

1. Dhingra PL. Common disorders of oral cavity: Diseases of ear, nose and throat (3rd ed). New Delhi: India, Elsevier 2004; 265-67.
2. Shimkhada, John W, Riti and Peabody. Tobacco control in India. Bulletin of the World Health Organization 2003;81:48-52.

3. Ahmad MS, Ali SA, Ali AS, et al. Epidemiological and etiological study of oral submucous fibrosis among gutkha chewers of Patna, Bihar, India. J Indian Soc Pedod Prev Dent 2006;24(2):84.

4. Naresh R Makwana, Viral R Shah, Sudha Yadav. A study on prevalence of smoking and tobacco chewing among adolescents in rural areas of Jamnagar District, Gujarat State. J Medical Sciences Research 2007;1:47-49.

5. Thangjam GS, Agarwal P, Khan I, et al. Transglutaminase-2 regulation by arecoline in gingival fibroblasts: J Dental Research Feb 2009;88(2):170-75.

6. Xia L, Tian You L, Yi Jun G, et al. Arecoline and oral keratinocytes may affect the collagen metabolism of fibroblasts. J Oral Pathol Med (E publication ahead of print) 2009.

7. Ariyawardana A, Athukorala AD, Arulanandam A. Effect of betel chewing, tobacco smoking and alcohol consumption on oral submucous fibrosis: A case-control study in Sri Lanka. J Oral Pathol Med 2006;35(4):197-201.

8. Kaushal V, Verma K, Manocha S, et al. Clinical evaluation of human placental extract (placentrex) in radiation-induced oral mucositis. International Journal of Tissue Reaction 2001;23(3):105-10.

9. Bywaters EGL, Holborow EJ, Keech MK. Hyaluronidase skin spreading effect, reconstitution of the dermal barrier to dye spread after Hyaluronidase injection. Br Med J 17 November, 1951;2(4741):1178-83.

10. Sinha SN, Jain PK. Intraoral injection of Hydrocortisone and placental extract in oral submucous fibrosis. Indian J Otolaryngology June 1978;30(2):103. 